NASA TECHNICAL NOTE

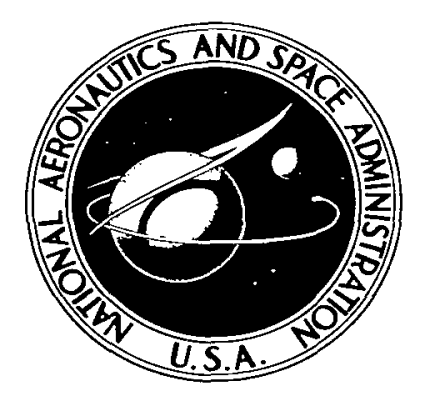

NASA IN D-3041

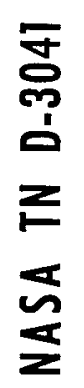

\title{
PERFORMANCE CORRELATION FOR ELECTRON-BOMBARDMENT ION SOURCES
}

\section{by Harold R. Kaufman \\ Lewis Research Center \\ Cleveland, Obio}

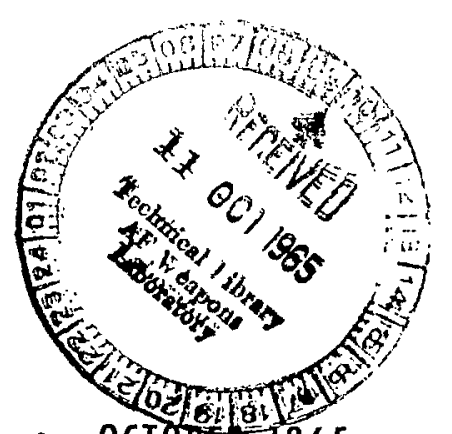

NATIONAL AERONAUTICS AND SPACE ADMINISTRATION - WASHINGTON, D. C. - OCTOBER H965 


\section{PERFORMANCE CORRELATION FOR ELECTRON-}

BOMBARDMENT ION SOURCES

By Harold R. Kaufman

Lewis Research Center

Cleveland, Ohio

\section{NATIONAL AERONAUTICS AND SPACE ADMINISTRATION}

For sale by the Clearinghouse for Federal Scientific and Technical Information Springfield, Virginia 22151 - Price $\$ 2.00$ 


\title{
PERFORMANCE CORRELATION FOR ELECTRON-BOMBARDMENT ION SOURCES
}

\author{
by Harold R. Kaufman \\ Lewis Research Center
}

\section{SUMMARY}

A procedure is presented for estimating the gross performance characteristics of a low-density electron-bombardment ion source. Although this ion source was initially investigated for use as a component of an electric-propulsion system, it should also be useful in a variety of other applications. The overall ion source performance was evaluated on the basis of two parameters; the minimum discharge energy (per ion), and the propellant utilization at a discharge energy twice this minimum value. The approach used was to derive generalizing parameters for both of these measures of performance by analysis of the more important ion-source processes. These generalizing parameters involve only overall ion-source measurements and readily available propellant properties. Within certain limits, the use of these generalizing parameters correlates the experimental data within about \pm 50 percent for minimum discharge energy and within about \pm 20 percent for the nominal propellant utilization (where the discharge energy is twice the minimum value). Two of these limits are that the discharge potential difference should not be less than about 2.0 times the first ionization potential of the propellant, and that the cyclotron radius of the primary electrons emitted from the cathode should not be more than about 0.45 times the ionization-chamber radius. This correlation, however, is not a substitute for good design. Careful handling of design details is still necessary if the best possible performance is to be obtained.

\section{INTRODUCTION}

The low-density electron-bombardment ion source of the type described in references 1 and 2 was developed as part of a broad electric-propulsion program, but its use is by no means limited to electric propulsion. Such ion sources can be used in many different ways. Other electron-bombardment sources used in scientific applications were characteristically developed sufficiently for the job at hand, and little information is available for other operating conditions (refs. 3 to 6 ). The extensive investigation of 
this electron-bombardment source for electric propulsion thus provides a unique body of information for prospective users. The use of this type of source is particularly convenient if the desired size and performance are close to those reported in the literature. If the contemplated ion source differs substantially from those units, though, the estimation of performance becomes much more difficult.

The ion-current capacity of the accelerator or extractor system has been investigated, and the results reported in references 7 and 8 . These investigations indicated, for example, that plates with hexagonal arrays of holes (with the open area equal to about half of the total area) make an efficient accelerator system when used with a centerline spacing about equal to the diameter of the holes. Based on the centerline spacing and the open area, the typical overall current capacity is from 0.7 to 1.0 times that indicated by Child's law. Operational limits must, of course, be observed. Frequent electrical breakdowns occur when the ratio of potential difference to gap spacing exceeds approximately 2000 volts per millimeter - at least for the surface finishes, ion impingement, and range of accelerator spacing ( 1 to $30 \mathrm{~mm}$ ) investigated for electric propulsion. The accelerator grid should be at a negative potential relative to the neutralizer and ion beam, the necessary amount being greater than about 15 percent of the total potential difference across the gap to prevent electron backstreaming and less than 40 to 50 percent to avoid large defocusing effects. The lifetime of an accelerator system has also been studied (ref. 9) and is primarily a function of chargeexchange erosion. For a lifetime of thousands of hours, such as will be necessary for electric propulsion, the average current density over the total accelerator area should be in the range of 10 to 50 amperes per square meter.

The selection of a cathode for the source is more of an art. For several hundreds of hours up to perhaps one thousand hours, a refractory-metal cathode is adequate (ref. 10). A refractory-metal cathode is particularly convenient if frequent exposure to air is a problem. For a lifetime of thousands of hours, an oxide-matrix type (refs. 11 and 12) should be suitable or, if a low-work-function propellant is used, the propellant can be used to provide a self-renewing cathode coating (the autocathode of ref. 13). A neutralizer, if needed, may be selected in a manner similar to that of the cathodes. Present experience indicates that a neutralizer that is shielded from direct ion impingement presents an erosion problem (from charge-exchange ions) no more difficult than that of the ionization-chamber cathode. While scaling must be mostly by trial and error, the range of electron emission from a tenth of an ampere to tens of amperes has been explored and appears practical.

An electromagnet, if used, is perhaps the simplest and most predictable component of this ion source. A permanent-magnet design of comparable weight is also possible (ref. 14).

The ion sour ce proper, or ionization chamber, poses the most difficult problem for 
a new application. While some scaling laws are known, such as keeping a constant ratio of cyclotron radius to chamber radius, they do not lead to completely similar performance for ionization chambers of different sizes (refs. 15 and 16). Even greater discrepancies have been found for operation with different propellants (ref. 17). Thus there is a need for correlating the major effects of geometry and propellant changes on ionization-chamber performance. Further, if this correlation is to be useful, it should be simple and easy to apply.

The objective of this report is to present a procedure for estimating the gross performance characteristics of electron-bombardment ionization chambers. The approach used was to derive generalizing parameters for the discharge energy and propellant utilization by analysis of the more important ion-source processes. For maximum utility, these parameters should involve only overall ion-source measurements and readily available propellant properties.

Experimental data from a number of investigations were plotted as functions of the generalizing parameters and the results of the analysis compared with the experimental performance. The analysis and the resulting correlations are intended primarily for singly ionized atomic propellants other than the low-ionization-potential alkali metals. There should, however, be some limited validity for atoms of low ionization potential and simple diatomic molecules.

All quantities - with the exception of ionization cross section, atomic or molecular mass, and energy per ion or neutral - are defined in mks units (similar to the SI or International System of units). The exceptions are in the frequently used units of $\pi \mathrm{a}_{\mathrm{o}}^{2}$, atomic mass units (amu), and electron-volts (eV). All symbols are defined in the appendix.

\section{ANALYSIS}

The purpose of this analysis is to derive parameters suitable for the correlation of electron-bombardment ion-source performance, and to indicate the approximate variation of ion-source performance with variations of these parameters. The overall performance of the electron-bombardment ion source, shown schematically in figure 1, can be measured in terms of the propellant supplied to the ion source, the electrical power expended, and the extracted ion beam. The acceleration process, of course, requires power. This power, however, is readily estimated (at near 100-percent efficiency) and is not a concern in this report. Neither are the heater powers - if any - for the cathode and neutralizer, or the power to sustain the magnetic field - if a permanent-magnet design is not used. Not only are these latter powers small compared with the discharge and acceleration powers in a well-designed ion source, but they also vary considerably with the specific designs selected. Thus only the discharge power (from the $\Delta V_{i}$ power supply in fig. 1) is considered in this analysis. (Even if the efficiency of the ion source 


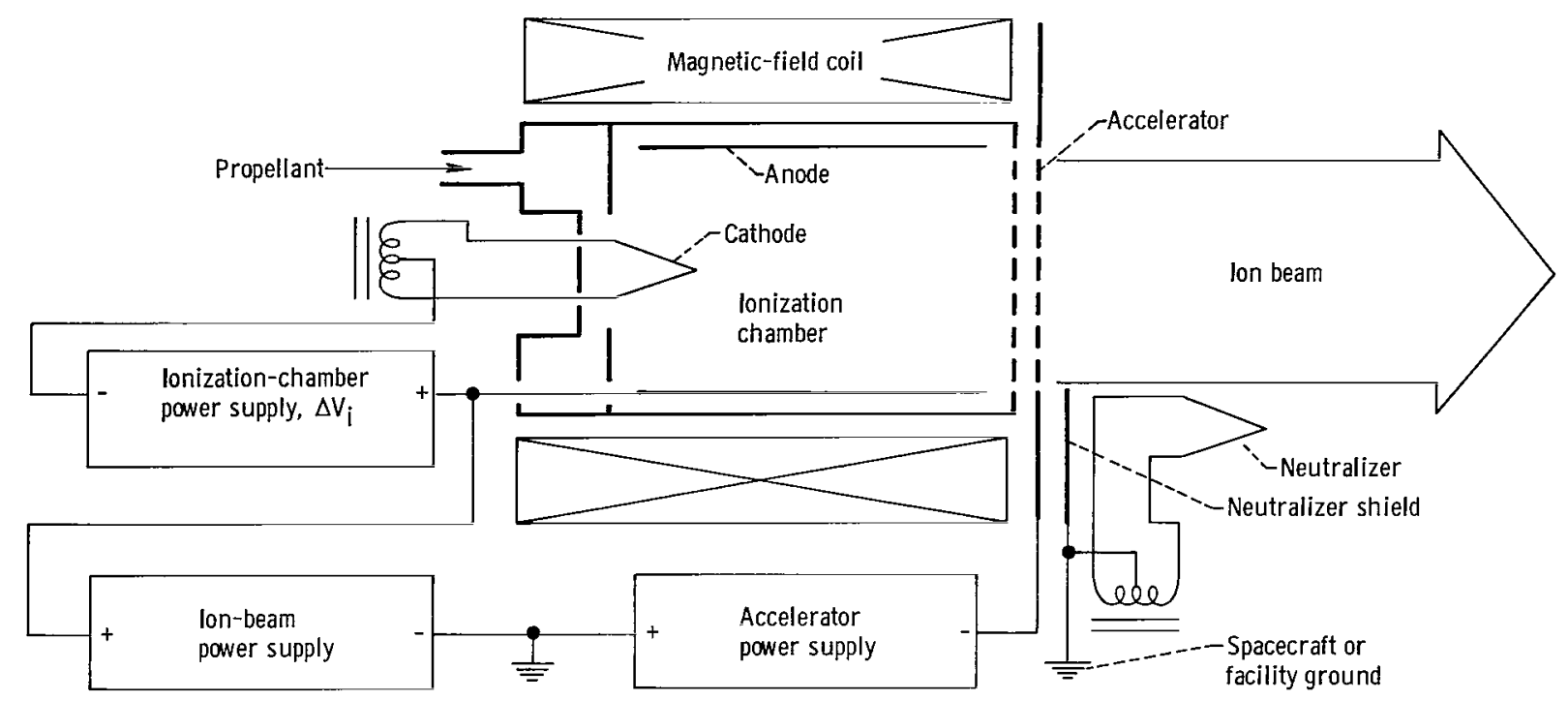

Figure 1. - Schematic diagram of ion source.

is considered to be relatively unimportant, as in most ground-based applications, the discharge process often limits the overall system performance. For example, poor ionization-chamber performance may push cathode requirements beyond the state of the art, or result in a complex cooling system to handle the large power losses. Also, the vacuum-pump capacity required for a given ion-beam current may be determined by the propellant utilization, or lack thereof.)

In the model assumed for the ionization chamber, the mean free paths for all collision processes are much greater than the ionization-chamber dimensions. A magnetic field is thus necessary to contain the high-energy ionizing electrons for a long enough time to have ionizing collisions. The plasma in the ionization chamber is dense, in that the Debye shielding distance is very small compared with the chamber dimensions. Plasma neutrality must therefore be observed. The radial potential difference in the plasma $\Delta V_{p}$ is generally small compared with the total ionization-chamber potential difference $\Delta \mathrm{V}_{\mathrm{i}}$. Thus most of the potential difference $\Delta \mathrm{V}_{\mathrm{i}}$ is found at the cathode sheath, and primary electrons from the cathode are injected into the ionization chamber with an energy of about $q \Delta V_{i}$. The electron energy distribution in the ionization chamber is far from equilibrium (refs. 18 and 19). The secondaries from the ionization process, together with some primaries from the cathode that have lost most of their energy, form a near-Maxwellian low-energy group. The higher energy primaries form another group that extends from low energy to the initial injection energy. This electron energy distribution is approximated in the analysis with two groups. The first group is assumed to be high-energy primaries at the initial injection energy, and the second group is assumed to be low-energy secondaries from the ionization process. This two-group model for the electron distribution is a reasonable approximation only 


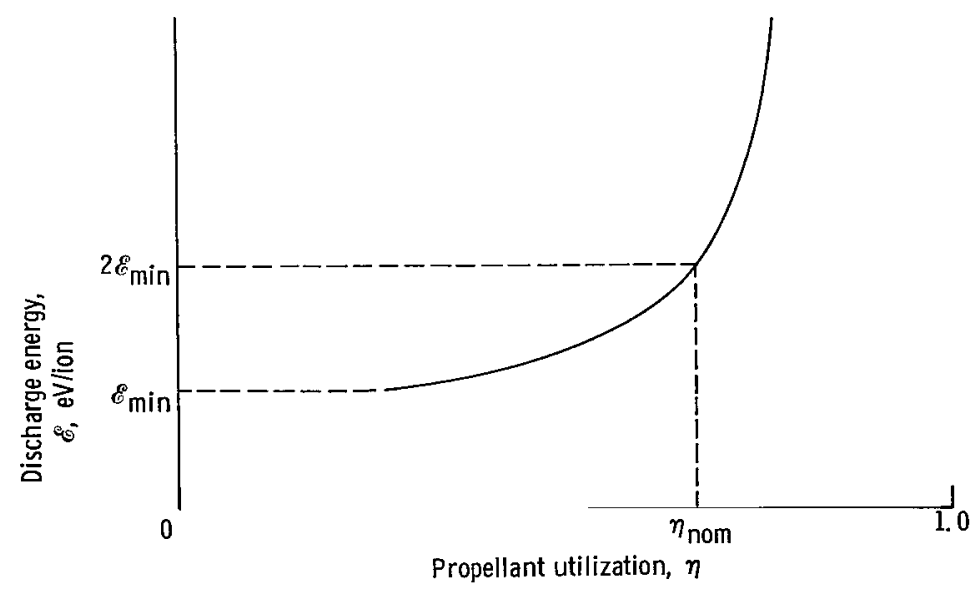

Figure 2. - Ionization-chamber performance.

if the product of the neutral density and the ionization cross section $n_{0} Q_{i}$ is substantially larger than the product of the electron density and the coulomb cross section $\mathrm{n}_{-} \mathbf{Q}_{\mathrm{c}^{*}}$. The rapid increase in coulomb cross section with decreasing electron energy therefore makes this model invalid at sufficiently low ionization-chamber potential differences. The electron distribution is also affected by the propellant utilization efficiency. For low utilizations, the high-energy primary electrons should be a small fraction of the total electron population, and the plasma properties should then be a function primarily of the secondary-electron density and energy. The primary-electron density must, of course, increase for higher propellant utilizations. The various assumptions made in this model will be discussed in more detail at appropriate places in the analysis.

The usual form in which the propellant flow rate, discharge power, and extracted ion current are presented is as a plot of discharge energy (per ion) against propellant utilization, as indicated in figure 2. The discharge energy is defined as the power from the $\Delta V_{i}$ power supply in figure 1 divided by the ion-beam current. Although there are some additional currents to consider, the current from the $\Delta V_{i}$ power supply is approximately equal to the emission from the cathode. The propellant utilization is defined as the fraction of the propellant flow that appears as ions in the extracted beam. The propellant flow is usually assumed to be a constant value for a plot, such as figure 2, so that each propellant utilization corresponds to a particular value of extracted ion current. The propellant flow is usually expressed as an equivalent current (the current that would result if each neutral atom or molecule carried one electronic charge). The shape of the energy-utilization curve (fig. 2) varies considerably with changes in ion-source geometry or operating conditions, so that it is not possible to predict the entire energyutilization relation with a simple analysis. Instead, two points have been selected as giving, by themselves, a fair indication of the curve shape. The first of these is the 
minimum discharge energy $\mathscr{E}_{\text {min }}$. As indicated in figure 2, there is usually little variation in discharge energy at low propellant utilizations, so that some extrapolation to low propellant utilizations may be used without introducing a large error into $\mathscr{E}_{\min }$.

In general, there is no maximum value of propellant utilization. Increasing the discharge power serves to increase continuously the propellant utilization, although at an ever-decreasing rate. The usual limitations are the practical ones of cathode emission and the discharge-power requirement. Thus some degree of arbitrariness is involved in selecting a representative propellant utilization. The value used herein is the propellant utilization corresponding to a discharge energy $\mathscr{E}$ that is twice the minimum discharge energy $\mathscr{E}^{\mathscr{E}}$. This utilization is called the nominal propellant utilization $\eta_{\text {nom }}$ and is indicated on the abscissa of figure 2. The analysis conducted in this section, then, is directed specifically at obtaining approximate mathematical expressions for the minimum discharge energy $\mathscr{E}_{\mathrm{min}}$ and the nominal propellant utilization $\eta_{\text {nom }}$. The generalizing parameters for $\mathscr{E}_{\min }$ and $\eta_{\text {nom }}$ can, in turn, be determined from the form of these mathematical expressions.

\section{Minimum Discharge Energy}

The derived form of the generalizing parameter will depend on which physical process is assumed to be most important and thus can be expected to control the similarity relation. The controlling process for the discharge energy was assumed to be the ohmic heating of the ionization-chamber plasma. As indicated in reference 1, efficient operation of this type of ion sour ce requires a small radial potential difference in the plasma. In contrast, inefficient operation of an electron-bombardment source can result from a large potential difference in the plasma, with most of the discharge energy going into ohmic heating of the plasma. The ohmic heating typically produces much excitation, but little ionization. The validity and limitations of this controllingprocess assumption will be discussed further in connection with the comparison of the analytical results and the experimental data.

A base or reference level $\mathscr{E}_{0}$ can be introduced and defined as the value of $\mathscr{E}$ that would be obtained with a negligible potential difference in the plasma $\Delta \mathrm{V}_{\mathrm{p}}$. The mini mum discharge energy $\mathscr{E}^{\mathscr{E}} \mathrm{min}$ (at conditions other than with negligible $\Delta V_{p}$ ) should thus be a function of $\xi_{o}$ and $\Delta V_{p} / \Delta V_{i}$. Specifically, the useful energy for ionization should vary as $\Delta \mathrm{V}_{\mathrm{i}}-\Delta \mathrm{V}_{\mathrm{p}}$. Thus the base level of discharge energy should be multiplied by the factor $\Delta V_{i} /\left(\Delta V_{i}-\Delta V_{p}\right)$, and

$$
\mathscr{E}_{\min }=\frac{\mathscr{E}_{\mathrm{o}}}{1-\frac{\Delta V_{p}}{\Delta V_{i}}}
$$


Most of the following analysis to determine $\mathscr{E}_{\min }$ is devoted to obtaining an expression for the radial potential difference $\Delta V_{p}$ in equation (1).

The radial potential variation in the plasma is assumed to be governed primarily by the mobility of the low-energy secondary electrons, since the primary electrons should constitute a small fraction of the total electron population at low propellant utilizations. There is, of course, one secondary electron produced for each ion extracted from the source. More ions are produced, however, than are extracted. Most of the ions that reach the downstream end of the ionization chamber are extracted from the source. The ions that go upstream, though, become neutralized at the upstream boundary of the ionization chamber. For simplicity, a number of ions equal to the number extracted will be assumed to go upstream. Ions will also be lost to the cylindrical side wall (or anode), but the radial potential gradient in the plasma reduces these losses. The total production rate of secondary electrons, then, is assumed to correspond to twice the ionbeam current. Assuming, for simplicity, a uniform plasma throughout a cylindrical ionization chamber of length $\ell_{i}$ and radius $r_{i}$ gives the differential form of Ohm's law

$$
\mathrm{dV}_{\mathrm{p}}=\frac{\mathrm{J}_{-} \mathrm{dr}}{2 \pi \mathrm{r} \ell_{\mathbf{i}_{1} \sigma_{1}}}
$$

with $V_{p}$ the plasma potential, $\sigma_{\perp}$ the radial conductivity across the magnetic field, and $\mathrm{J}_{-}$the secondary-electron current produced within the radius $r$. As already indicated, this electron current can be expressed in terms of the ion-beam current density $j_{+}$(the average value over the total accelerator area):

$$
J_{-}=2 \pi r^{2} j_{+}
$$

Using equation (3) gives equation (2) in the form

$$
\mathrm{dV}_{\mathrm{p}}=\frac{\mathrm{j}_{+} \mathrm{r} \mathrm{dr}}{\ell_{\mathrm{i}} \sigma_{\perp}}
$$

Integrating $v_{p}$ from 0 to $V_{p}$ and $r$ from 0 to $r_{i}$ yields

$$
\Delta V_{p}=\frac{j_{+} r_{i}^{2}}{2 \ell_{i} \sigma_{\perp}}
$$

For the conductivity $\sigma_{1}$, a simplified approach - in which all the secondary electrons are assumed to be moving at the same velocity - should be adequate for the 
approximate result required herein. The root-mean-square ( $\mathrm{rms}$ ) value was used for that velocity. The $\mathrm{rms}$ velocity of an electron is

$$
\bar{v}_{-}=\left(\frac{3 q \bar{v}}{m}\right)^{1 / 2}=7.27 \times 10^{5} \bar{v}_{-}^{1 / 2}
$$

with $\overline{\mathrm{V}}$ the electron temperature in volts. (The equivalent expression with the temperature in ${ }^{\circ} \mathrm{K}$ can be obtained from eq. (6) by substituting the product $k T$ for $q \bar{V}$.) $T$ he symbols $\mathrm{q}$ and $\mathrm{m}$ are the electronic charge and mass of an electron. The rms electron velocity is used to obtain the conductivity of a plasma in the absence of a magnetic field (given in similar nomenclature in ref. 20):

$$
\sigma_{\mathrm{o}}=\frac{\ell_{-} \mathrm{n}_{-} \mathrm{q} \overline{\mathrm{v}}_{-}}{3 \overline{\mathrm{V}}}=3.88 \times 10^{-14} \frac{\ell_{-} \mathrm{n}}{\overline{\mathrm{V}}_{-}^{1 / 2}}
$$

The quantities $\ell_{-}$and $\mathrm{n}_{-}$are the electron mean free path and density. The strongfield approximation for electron conductivity normal to a magnetic field, which is appropriate for electron motion in an ionization chamber, is

$$
\sigma_{1}=\frac{\sigma_{0}}{\omega_{-}^{2} \tau_{-}^{2}}
$$

The product of electron cyclotron frequency and collision time $\omega \tau$ may be replaced by the ratio of electron mean free path to cyclotron radius $\ell \sqrt{\bar{r}_{c}}$ (note that $\ell$ equals $\tau_{-} \bar{v}_{-}$, and $\bar{r}_{c}$ equals $\left.\bar{v}_{-} / \omega_{-}\right)$:

$$
\sigma_{1}=\sigma_{o}\left(\frac{\bar{r}_{c}}{\ell_{-}}\right)^{2}=3.88 \times 10^{-14} \frac{\mathrm{n}_{-} \overline{\mathrm{r}}_{\mathrm{c}}^{2}}{\ell_{-} \mathrm{V}_{-}^{1 / 2}}
$$

The cyclotron radius of an electron with a velocity $v_{-}$is

$$
r_{\mathrm{c}}=\frac{\mathrm{mv}}{\mathrm{Bq}}
$$

Using the rms velocity $\bar{v}_{-}$from equation (6) to substitute for $v_{-}$gives the cyclotron radius in terms of $\bar{V}_{-}$: 


$$
\overline{\mathrm{r}}_{\mathrm{c}}=\frac{\left(3 \mathrm{~m} \overline{\mathrm{V}}_{-}\right)^{1 / 2}}{\mathrm{~Bq}^{1 / 2}}=4.13 \times 10^{-6} \frac{\overline{\mathrm{V}}_{-}^{1 / 2}}{\mathrm{~B}}
$$

The conductivity in the absence of a magnetic field given by equation (7) is only about one-third of the value given in reference 21 for a Maxwellian electron-velocity distribution. This difference is due mostly to the fact that the high-velocity portion of the Maxwellian distribution is responsible for most of the conduction. For the strongmagnetic-field case (which is the one of interest for an ionization chamber), the simplified approach of equation (9) is within about 10 percent of the corresponding Maxwelliandistribution approach. Thus equation (9) for $\sigma_{\perp}$ is sufficiently accurate to use in the plasma potential difference expression, equation (5). The variables of equation (9), though, are not suitable for a generalizing parameter based on overall measurements and readily available properties. Equivalent expressions for the electron mean free path, density, and temperature will therefore be obtained. The electron cyclotron radius is not particularly easy to obtain either, but it is useful for the physical interpretation of results when expressed as a ratio with the ionization-chamber radius.

The minimum radial potential difference in the plasma $\Delta V_{p}$ is obtained at low propellant utilizations, where collisions with neutrals are the dominant factor in determining the electron mean free path. For the uniform-electron-velocity approach used herein,

$$
\ell_{-}=\frac{1}{\mathrm{n}_{\mathrm{O}} \mathrm{Q}_{\mathrm{O}}}
$$

The neutral density $\mathrm{n}_{\mathrm{o}}$ can be determined from the neutral velocity, the neutral flow rate, and the ionization-chamber configuration. The rms neutral velocity is

$$
\overline{\mathrm{v}}_{\mathrm{o}}=\left(\frac{3 \mathrm{kT}}{\mathrm{M}}\right)^{1 / 2}
$$

The typical projected-area blockage of the accelerator system is about half of the total area (ref. 7). This blockage is used as an estimate of the flow obstruction, along with a neutral equivalent-current density $j_{0}$ (based on the ionization-chamber cross section, which equals the accelerator total area), to obtain the neutral density upstream of the 
accelerator system from free-molecular-flow theory. The neutral density is thus approximately $^{1}$

$$
\mathrm{n}_{\mathrm{o}}=\frac{6.52 \mathrm{j}_{\mathrm{o}}}{q \overline{\mathrm{v}}_{\mathrm{o}}}
$$

Variations of neutral density within the ionization chamber were ignored, so that equation (14) was assumed to apply throughout the chamber. Using equation (13) for the neutral velocity $\bar{v}_{o}$ and assuming a typical neutral temperature of $500^{\circ} \mathrm{K}$ (the neutral temperature is primarily a function of the ionization-chamber wall temperature) result in the evaluation of the neutral density as

$$
\mathrm{n}_{\mathrm{o}}=2.83 \times 10^{29} \mathrm{j}_{\mathrm{o}} \mathrm{M}^{1 / 2}
$$

Finally, if the neutral mass is expressed in the more-convenient atomic mass units (amu) of $W$, equation (15) becomes

$$
\mathrm{n}_{\mathrm{o}}=1.15 \times 10^{16} \mathrm{j}_{\mathrm{o}} \mathrm{W}^{1 / 2}
$$

The use of a 50-percent blockage for neutrals might indicate to the reader that a similar blockage should have been used for ions reaching the downstream end of the ionization chamber. The local electric field, though, tends to direct the ions around the grids, so that the effective blockage for ions is much less than that indicated by the projected-area blockage.

To evaluate $Q_{O}$, the secondary-electron temperature is needed. For most atoms the excitation of neutrals limits the secondary-electron temperature $\bar{V}$ in a plasma with a fair number of neutrals to roughly half the first ionization potential $\varphi_{\mathbf{i}}$ (refs. 13, 18, and 19). A secondary-electron temperature of $\varphi_{i} / 2$ will therefore be assumed in this analysis, which corresponds to an rms electron energy of $3 \varphi_{i} / 4$. Less data are generally available for the electron-neutral collision cross section $Q_{0}$ than for the maximum cross section for single ionization $Q_{\mathbf{i}}$. Since $Q_{\mathbf{i}}$ is also required for the subsequent analysis of nominal utilization, expressing $Q_{0}$ in terms of $Q_{\mathbf{i}}$ is preferable

$1_{\text {The constant }} 6.52$ was obtained by using the current density in a single hole as a starting point. The neutral density far upstream from a single hole is given by a constant of 4.34 in eq. (14). Making allowance for a 50-percent blockage in the flow area would increase the constant to 8.68. Immediately upstream of the grid, the density of neutrals moving in the downstream direction is the same as it is far upstream. The density of neutrals moving upstream at that location, though, is cut in half - as a result of the 50-percent blockage. The density immediately upstream of the grid is thus given by a constant that is 25 percent less than 8.68 - or 6.52 . 
to using $Q_{o}$ directly. From reference 22, the value of $Q_{0}$ at an electron energy of $3 \varphi_{i} / 4$ varies from about 4 to 8 times $Q_{i}$ for a number of gases. ${ }^{2} A$ mean value of $6 Q_{i}$ will therefore be used in place of $Q_{0^{*}}$ To avoid large negative exponents, $Q_{i}$ will be in units of $\pi a_{0}^{2}$. With the proper substitutions for $n_{0}$ and $Q_{0}$, equation (12) becomes

$$
\ell_{-}=\frac{1.15 \times 10^{3}}{j_{0} Q_{i} W^{1 / 2}}
$$

The secondary-electron density is, of course, close to the ion density $\mathbf{n}_{+^{*}}$ The latter can be estimated from Bohm's criteria for a stable plasma sheath. According to this criterion (ref. 23), the ions arriving at a sheath must have a directed velocity equivalent to at least half of the electron temperature. Using $\bar{V}_{-} / 2$, with $\bar{V}_{-}$equal to $\varphi_{i} / 2$, gives the ion drift velocity toward the ends of the ionization chamber

$$
\mathrm{v}_{+}=\left(\frac{\mathrm{q} \varphi_{\mathrm{i}}}{2 \mathrm{M}}\right)^{1 / 2}
$$

where $M$ is the ion mass. The ion density in the ionization chamber, then, can be approximated with the ion-beam current density $j_{+}$divided by the product of electronic charge and ion velocity $\mathrm{qv}_{+}$:

$$
\mathrm{n}_{+}=\frac{\mathrm{j}_{+}}{\mathrm{qv_{+ }}}
$$

Using equation (18) for the ion velocity gives equation (19) in the form

$$
\mathrm{n}_{+}=2.20 \times 10^{28} \frac{\mathrm{j}_{+} \mathrm{M}^{1 / 2}}{\varphi_{\mathrm{i}}^{1 / 2}}
$$

Or, in terms of the mass $\mathrm{W}$ in amu,

$$
n_{+}=8.99 \times 10^{14} \frac{j_{+} w^{1 / 2}}{\varphi_{i}^{1 / 2}}
$$

${ }^{2}$ Atomic hydrogen, $\mathrm{H}_{1}$; diatomic hydrogen, $\mathrm{H}_{2}$; helium, He; diatomic nitrogen, $\mathrm{N}_{2}$; diatomic oxygen, $\mathrm{O}_{2}$; neon, $\mathrm{Ne}$; argon, $\mathrm{A}$; krypton, $\mathrm{Kr}$; xenon, $\mathrm{Xe}$; and mercury, $\mathrm{Hg}$. 
Using equation (17) for $\ell_{-}$, equation (21) for $\mathrm{n}_{-}$(assumed equal to $\mathrm{n}_{+}$), and $\varphi_{\mathrm{i}} / 2$ for $\bar{V}_{-}$gives equation (9) as

$$
\sigma_{\perp}=2.99 \times 10^{-2} \frac{\mathrm{j}_{\mathrm{O}_{+}} \mathrm{Q}_{\mathrm{i}} \overline{\mathrm{r}}_{\mathrm{c}}^{2} \mathrm{~W}}{\varphi_{\mathrm{i}}}
$$

Using this conductivity gives equation (5) in the form

$$
\Delta V_{p}=\frac{16.7 \varphi_{i}}{j_{o} \ell_{i} Q_{i} W}\left(\frac{r_{i}}{\bar{r}_{c}}\right)^{2}
$$

Note that the ion-beam current density $j_{+}$does not appear in the preceding equation. Thus any small value of $\mathbf{j}_{+}$is reasonable as long as the electron-neutral collisions dominate and the neutral density is not appreciably depleted.

The plasma potential difference $\Delta \mathrm{V}_{\mathrm{p}}$ appears in equation (1) only in the ratio $\Delta V_{p} / \Delta V_{i}$. Using a typical value of $4 \varphi_{i}$ for $\Delta V_{i}\left(4 \varphi_{i}\right.$ is generally near the electron energy for maximum single-ionization cross section $Q_{i}$ ). The ratio of plasma potential difference to discharge potential difference is

$$
\frac{\Delta V_{p}}{\Delta V_{i}}=\frac{4.18}{j_{o}{ }_{i} Q_{i} W}\left(\frac{r_{i}}{\bar{r}_{c}}\right)^{2}
$$

Using equation (24) in equation (1) and rounding off the numerical constant to correspond to the accuracy of the analysis give

$$
\mathscr{E}_{\min }=\frac{{ }_{0}^{E_{o}}}{1-\frac{4\left(\mathrm{r}_{\mathrm{i}} / \overline{\mathrm{r}}_{\mathrm{c}}\right)^{2}}{j_{\mathrm{o}} \ell_{i} \mathrm{Q}_{\mathrm{i}} \mathrm{W}}}
$$

To estimate $\mathscr{E}_{0}$, it is noted that a typical ionization energy for high-energy electrons is $30 \mathrm{eV}$ per ion. The relative increase in excitation cross section compared with the ionization cross section at low values of electron energy (near $4 \varphi_{j}$ ) would tend to increase this energy. Thus, perhaps $50 \mathrm{eV}$ per ion might be a reasonable base level 
for ion production in an ionization chamber. With only half the ions escaping into the beam, the value of $\mathscr{E}_{\mathrm{o}}$ in equation (25) might be estimated at about $100 \mathrm{eV}$ per ion. This value will be used in the initial comparison with experimental discharge-energy data.

As discussed in this derivation, the $\mathrm{rms}$ velocity is used for secondary electrons. When equation (11) is expressed in terms of the assumed secondary-electron temperature of $\varphi_{i} / 2$, the rms electron cyclotron radius to be used in equation (25) becomes

$$
\overline{\mathrm{r}}_{\mathrm{c}}=2.92 \times 10^{-6} \frac{\varphi_{\mathrm{i}}^{1 / 2}}{\mathrm{~B}}
$$

To summarize this derivation, the controlling process was assumed to be ohmic heating of the ionization-chamber plasma. The other major assumptions made were

(1) A neutral temperature of $500^{\circ} \mathrm{K}$

(2) A 50-percent accelerator blockage as the only neutral-flow restriction

(3) The discharge potential difference equal to 4 times the first ionization potential of the propellant

(4) Equal numbers of ions going to the two ends of the ionization chamber, with only the downstream ions being extracted

(5) Directed ion velocities toward the ends of the chamber in accord with Bohm's criteria for a stable plasma sheath

(6) The motion of secondary electrons dominated by collisions with neutrals

(7) The excitation of neutrals limits the secondary-electron temperature to the equivalent of half the first ionization potential

(8) All secondary electrons moving at rms velocity

(9) An electron-neutral collision cross section equal to 6 times the maximum cross section for single ionization

(10) Uniform ion production rate, particle densities, and particle energies throughout the ionization chamber

\section{Nominal Propellant Utilization}

Since an approximate analytical expression for the minimum discharge energy $\mathscr{E}^{\mathscr{E}}$ min has been derived, an equivalent analysis for the nominal propellant utilization $\eta_{\text {nom }}$ is in order. The propellant utilization is assumed to be the integrated probability of ionization $p_{i}$ for neutrals passing through the portion of the ionization chamber in which beam ions are produced. The incremental probability for low propellant utilizations $\left(\eta_{\text {nom }}<<1\right)$ is simply the product of the electron-neutral ionizing cross section, the neutral residence time, the velocity of the ionizing electrons, and the incremental density 
of the ionizing electrons - or $Q_{i} t_{o} v_{-} d n_{-}$. The multiplication by the factor $1-p_{i}$ is necessary for the integration to propellant utilizations near unity. In effect, the ions that are the result of integration to any propellant utilization must be subtracted from the remaining neutrals - since ionized neutrals cannot be re-ionized. The differential equation for probability of ionization, then, is

$$
d p_{i}=Q_{i} t_{0} v_{-}\left(1-p_{i}\right) d n
$$

Integrating $\mathrm{p}_{\mathrm{i}}$ from 0 to $\eta_{\text {nom }}$ and $\mathrm{n}_{-}$from 0 to $\mathrm{n}_{-}$results in

$$
\eta_{\text {nom }}=1-\exp \left(\mathrm{n}_{-} \mathrm{Q}_{\mathrm{i}} \mathrm{t}_{\mathrm{o}} \mathrm{v}_{-}\right)
$$

The major assumptions for the preceding integration are uniformity of electron density and energy throughout the ionization chamber. Also, all neutrals should have the same residence time. The variables of equation (28) are not suitable for a simple generalizing parameter. With the exception of $Q_{i}$, which will again be expressed in units of $\pi a_{0}^{2}$, these variables should be replaced by others that are easier to obtain.

The ionizing electrons are, of course, the high-velocity primaries that are emitted from the cathode and accelerated through the cathode sheath to an energy of about $4 \varphi_{i}$. The density of these ionizing electrons is small at low propellant utilizations and must be increased to obtain higher utilizations. The increase in discharge energy (per ion) that accompanies any substantial increase in utilization is due, in part, to the accompanying increase in plasma potential difference $\Delta \mathrm{V}_{\mathrm{p}}$. The increase in $\Delta \mathrm{V}_{\mathrm{p}}$ is, in turn, partly due to the ionizing electrons becoming a greater fraction of the total electron population. The associated decrease in neutral density will tend to increase the electron mean free path and thus, as indicated by equation (9), also tend to increase $\Delta \mathrm{V}_{\mathrm{p}}$.

As a result of the variety of operating conditions that can exist, doubling the discharge energy (to $2{ }^{E} \mathrm{~min}$ ) does not correspond to the ionizing electrons reaching any fixed fraction of the electron (or ion) population. For the approximate approach used herein, and for the lack of a better relation, it will be assumed that the primary-electron density at the nominal propellant utilization equals half the ion density of equation (21):

$$
n_{-}=4.50 \times 10^{14} \frac{j_{+} w^{1 / 2}}{\varphi_{i}^{1 / 2}}
$$

Assuming that the extracted ions come only from the downstream half of the ionization chamber results in the average residence time for a neutral in that half: 


$$
t_{0}=\frac{\frac{l_{i}}{2} n_{0} q}{j_{o}}
$$

Using equation (16) for $n_{0}$ gives the average time available for ionization:

$$
t_{o}=9.21 \times 10^{-4} \ell_{i} \mathrm{~W}^{1 / 2}
$$

The ionizing electron velocity is, of course,

$$
v_{-}=\left(\frac{2 q V_{-}}{m}\right)^{1 / 2}
$$

With the substitution of $4 \varphi_{\mathbf{i}}$ for the electron potential $\mathrm{V}_{-}$,

$$
\mathrm{v}_{-}=\left(\frac{8 \mathrm{q} \varphi_{\mathrm{i}}}{\mathrm{m}}\right)^{1 / 2}=1.19 \times 10^{6} \varphi_{\dot{i}}^{1 / 2}
$$

Using equation (29) for $n_{-}$, equation (31) for $t_{0}$, equation (33) for $v_{-}, Q_{i}$ in $\pi a_{0}^{2}$ units, and with the numerical constant appropriately rounded off gives equation (28) as

$$
\eta_{\text {nom }}=1-\exp \left(-0.004 j_{+} \ell_{i} Q_{i} w\right)
$$

To summarize the derivation for the nominal propellant utilization, the controlling process was assumed to be the probability that a neutral would be ionized by primary electrons in the portion of the ionization chamber in which beam ions are produced. The further assumptions, in addition to those mentioned for the derivation of minimum discharge energy, were

(1) All primary electrons are at an energy equivalent to the total discharge potential difference

(2) The ionization cross section for neutrals is equal to the maximum value for single ionization

(3) All beam ions come from the downstream half of the ionization chamber

(4) The primary-electron density is equal to half the ion density indicated by Bohm's criteria and the secondary-electron temperature 


\section{Generalizing Parameters}

With the number of approximations and assumptions that are involved in the derivations, accurate numerical estimates should not be expected. The functional relations implied by equations (25) and (34), though, may have significance in correlating experimental results. The groupings of independent variables in these equations are $j_{o} \ell_{i} Q_{i} W\left(\bar{r}_{c} / r_{i}\right)$ and $j_{+} \ell_{i} Q_{i} W$. Equations (25) and (34) can be rewritten as general functions of these groupings:

$$
\begin{gathered}
\mathscr{E}_{\min }=\mathrm{f}\left[\mathrm{j}_{\mathrm{o}} \ell_{\mathrm{i}} \mathrm{Q}_{\mathrm{i}} \mathrm{W}\left(\mathrm{r}_{\mathrm{c}} / \mathrm{r}_{\mathrm{i}}\right)^{2}\right] \\
\eta_{\text {nom }}=\mathrm{f}\left(\mathrm{j}_{+} \ell_{\mathrm{i}} \mathrm{Q}_{\mathrm{i}} \mathrm{W}\right.
\end{gathered}
$$

If the controlling processes have been assumed correctly, and if the other assumptions are at least qualitatively correct, then the functional relations (35) and (36) should be valid - even if the numerical results of equations (25) and (34) are not; that is, data for ${ }^{\mathscr{E}} \min$ and $\eta_{\text {nom }}$ from a variety of source geometries and operating conditions should correlate when plotted against the respective parameters $j_{0} \ell_{i} Q_{i} W\left(\bar{r}_{c} / r_{i}\right)^{2}$ and $j_{+} \ell_{i} Q_{i} W$. Such correlations are presented in the next section.

\section{COMPARISON WITH EXPERIMENT}

The parameters derived in the preceding section are used to correlate experimental data in this section. Before presenting the correlations, though, the sources and accuracy of the experimental data should be discussed.

\section{Sources and Accuracy of Data}

The maximum-cross-section data for single ionization were obtained from reference 24 for the propellants used herein. These cross sections, together with the corresponding atomic weights and first ionization potentials are shown in the following table: 


\begin{tabular}{|c|c|c|c|}
\hline Propellant & $\begin{array}{c}\text { Neutral } \\
\text { mass, } \\
\text { W, } \\
\text { amu }\end{array}$ & $\begin{array}{l}\text { First ioniza- } \\
\text { tion potential } \\
\qquad \begin{array}{c}\varphi_{\mathbf{i}} \\
\mathrm{eV}\end{array}\end{array}$ & $\begin{array}{c}\text { Maximum cross } \\
\text { section for first } \\
\text { ionization, } \\
\mathrm{Q}_{\mathrm{i}}, \\
\pi \mathrm{a}_{\mathrm{o}}^{2}\end{array}$ \\
\hline Atomic hydrogen & 1.0 & 13.6 & 0.8 \\
\hline Diatomic hydrogen & 2.0 & 15.6 & 1. 1 \\
\hline Atomic nitrogen & 14.0 & 14.5 & 1.5 \\
\hline Diatomic nitrogen & 28.0 & 15.5 & 2.8 \\
\hline Argon & 39.9 & 15.8 & 4.0 \\
\hline Krypton & 82.9 & 14.0 & 5.5 \\
\hline Cesium & 132.8 & 3.9 & 13.0 \\
\hline Mercury & 200.6 & 10.4 & 6.5 \\
\hline
\end{tabular}

Faired values of experimental ionization-chamber performance data were obtained from references $1,13,16,17,25$, and 26. Considerable unpublished data from past experiments at the Lewis Research Center were also used. The discharge-energy and propellant-utilization data were generally accurate within \pm 5 percent. Uncertainties in fairing data and poor data reproducibility increased the error to \pm 20 or 30 percent in several of the very low utilization points. All mercury propellant data were corrected for multiple ionizations (by reducing the propellant utilization) using the mean variation of the experimental data in reference 27, although the correction was usually within the cited uncertainty. Multiple ionizations at high discharge potential differences in propellants other than mercury caused an additional uncompensated error. The performance at such conditions, though, was generally so poor that the effect of multiple ionizations was relatively unimportant. The ions for the diatomic gases investigated were approximately 80 percent diatomic by a mass spectrometer in the ion beam. Small differences in the assumed percentages of diatomic and monatomic ions had little effect on the degree of correlation, so that assumed percentages of diatomic and monatomic ions of 80 and 20 percent were considered sufficiently accurate. For higher propellant utilizations than are presented herein for diatomic gases, the percentage of monatomic ions would be expected to increase.

For magnetic-field strength, the axial location with the minimum value in the ionization chamber was used. This location of minimum-magnetic-field strength was almost always immediately upstream of the accelerator grids. When the radial variation at this minimum-strength location was significant, a mean value of the field was used.

Despite the various sources of experimental error, the major cause of data variation was probably not error, but detailed differences in ion-source geometry. Examples of such differences are the various modes of propellant injection investigated in reference 28, the variation of magnetic-field shape used in reference 29, and the details of the various accelerator systems tested in reference 7. These differences can result in 


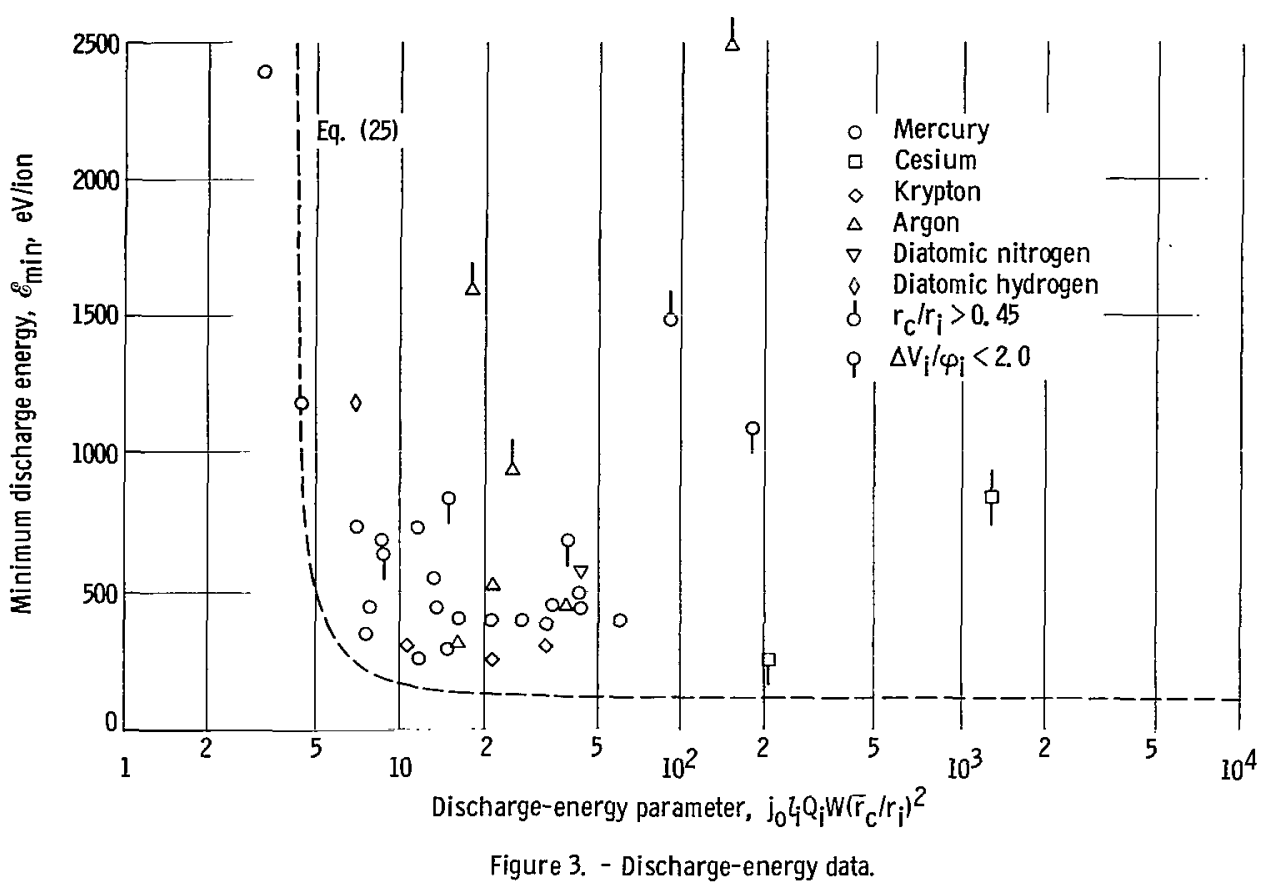

a \pm 50 -percent variation in discharge energy and a \pm 20 -percent variation in propellant utilization at otherwise good operating conditions.

\section{Correlation of Data}

The minimum-discharge-energy data are plotted as a function of the dischargeenergy parameter in figure 3. The agreement with the predicted variation of equation (25) is, at first glance, rather poor. (The estimated discharge energy of $100 \mathrm{ev} / \mathrm{ion}$ was used as the base level of discharge energy $\mathscr{E}_{0}$ in eq (25).) Examination of the data with high discharge energies, however, is revealing. Almost all such data that are far above the predicted trend have discharge potential differences $\Delta V_{i}$ that are less than 2. 0 times the first ionization potential $\varphi_{i}$, or a cyclotron radius for the primary electrons $r_{c}$ that is greater than 0.45 times the ionization-chamber radius $r_{i}$. (The primary-electron cyclotron radius can be determined from eq. (10) and eq (32), with $\mathrm{V}_{\text {- }}$ replaced by $\Delta \mathrm{V}_{\mathrm{i}}$ :

$$
\mathrm{r}_{\mathrm{c}}=3.37 \times 10^{-6} \frac{\Delta \mathrm{V}_{\mathrm{i}}^{1 / 2}}{\mathrm{~B}}
$$

Considering the critical nature of the $r_{c} / r_{i}$ limit and the ease of calculating $r_{c}$, the 
actual value of $\Delta \mathrm{V}_{\mathbf{i}}$ is preferred over the approximation $4 \varphi_{\mathbf{i}}$ ) Thus the greater discharge energies of these data correspond to either primary-electron energies well below the optimum for ionization cross section, or cyclotron radii for the primary electrons that are large enough to permit many of them to reach the anode without collisions. (The critical value might be expected to be 0.50 instead of 0.45 , but the finite size of the cathode makes the latter value more appropriate.)

If the tailed symbols in figure 3 are ignored, there is still a substantial discrepancy between the general trend of the data and equation (25). The higher values for the experimental data indicate that other losses, other than those considered in the dischargeenergy analysis, are significant. Guesses can be made as to the sources of these additional losses (such as ions reaching the anode and plasma turbulence increasing the loss of primary electrons to the anode), but present knowledge does not permit more than a guess.

The cesium data in figure 3 constitute a special case. Cesium is the only alkalimetal propellant investigated, and the electron-neutral collision cross section for such propellants averages about 40 times the maximum ionization cross section (from sodium, potassium, rubidium, and cesium data in ref. 22) - instead of 6 times, as assumed in the analysis. The model assumed in the preceding section also had two groups of electrons in the ionization chamber, with the ionizations produced almost entirely by the high-velocity primary electrons. This model is justified in that a primary electron normally has a high probability of having an ionizing collision with a neutral before it loses a substantial amount of energy to the secondary electrons by coulomb collisions. The low discharge potential differences used with cesium, though, reverses the probable mechanism of primary-electron energy loss; that is, a primary electron in a low $\Delta V_{i}$ cesium discharge has a high probability of losing most of its energy to the general electron population before having an ionizing collision. Most of the ionization in this type of cesium discharge, then, is provided by the "Maxwellian tail" of the general electron distribution, instead of by primary electrons. This would be a very inefficient ionization process for most propellants, but the low ionization potential of cesium keeps the discharge energy low. The basic difference between the usual discharge process and the discharge process with cesium (or probably any other low-ionization-potential propellant) at a low value of $\Delta \mathrm{V}_{\mathbf{i}}$, however, makes the use of the derived generalization parameters questionable. It would be interesting to compare the effects of high and low values of $\Delta \mathrm{V}_{\mathbf{i}}$, but sufficient data to obtain $\mathscr{E}_{\min }$ and $\eta_{\text {nom }}$ for a $\Delta V_{i}$ greater than $2 \varphi_{i}$ are not available for cesium. In any event, the low values of $\Delta \mathrm{V}_{i}$ used with cesium do not have the same significance that they would have with higher ionization-potential propellants.

For the same value of discharge-energy parameter, low-ionization-potential propellants might be expected to have lower discharge energies than other propellants. From 


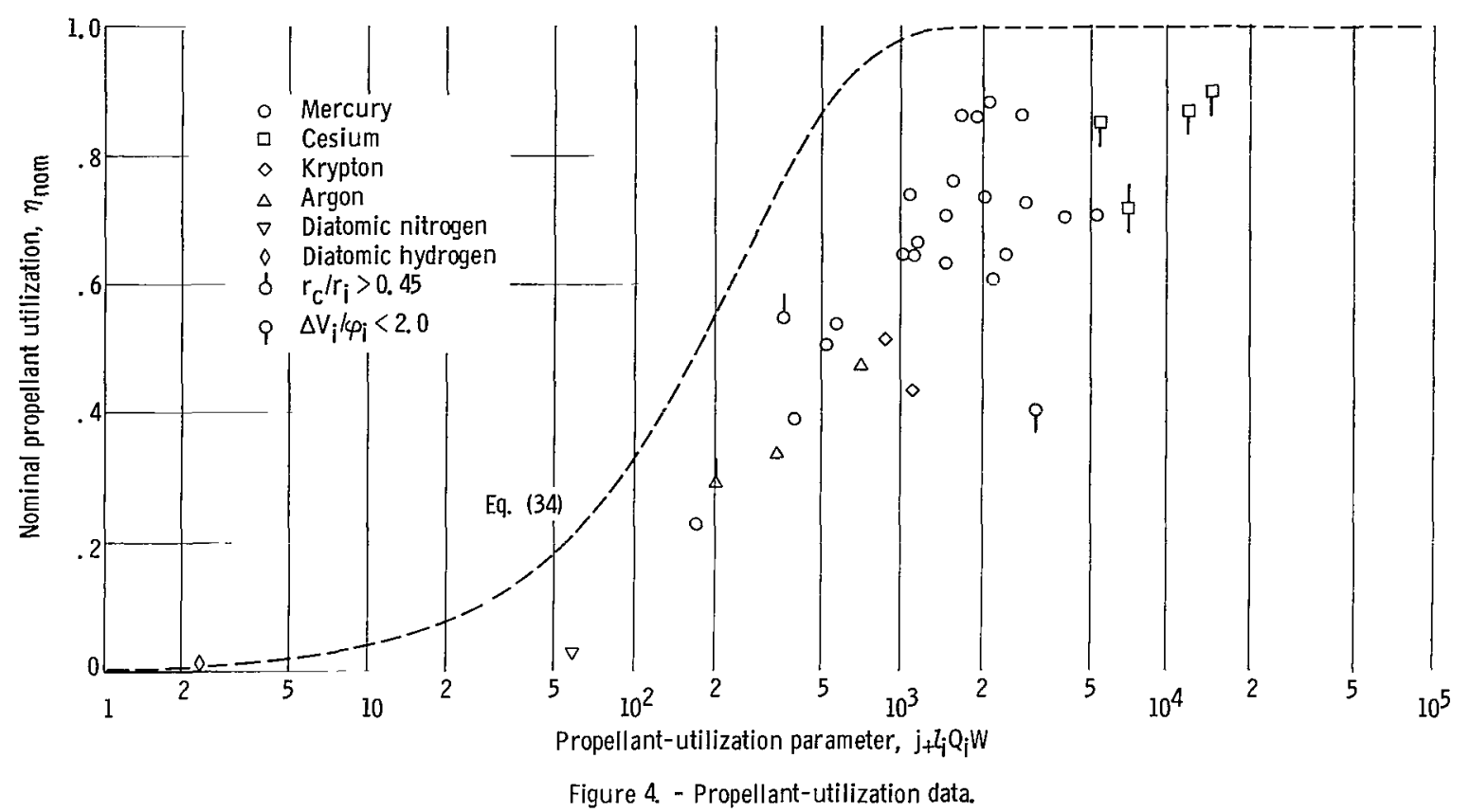

the data in figure 3 , though, no significant effect of ionization potential is apparent. The explanation might be that a higher fraction of the discharge energy is generally expended on excitation in low-ionization-potential propellants. This explanation is partly supported by the data on ion production by very high-energy electrons in reference 30 . The energy per ion drops with decreasing ionization potential in reference 30 , but at a much less than linear rate. Regardless of the explanation, the data of figure 3 indicate that the best simple correlation for all propellants is obtained by ignoring any possible effect of propellant ionization potential on experimental discharge energy.

The nominal propellant utilization data are plotted as a function of the propellant utilization parameter in figure 4. In this plot, only one point (other than with cesium propellant) has a $\Delta \mathrm{V}_{\mathrm{i}} / \varphi_{\mathrm{i}}$ less than 2.0 , but that point indicates that a low discharge potential difference also has an adverse effect on nominal propellant utilization. (It should be mentioned that the data in fig. 4 do not necessarily correspond to those in fig. 3. Some ionization-chamber data were available over a considerable but low range of $\eta$. Thus $\mathscr{E}_{\min }$ could be available without a corresponding value of $\eta_{\text {nom. }}$. The converse occurred when $\mathscr{E}$ rose very rapidly at high values of $\eta$. Under these conditions, even a rough estimate of $\mathscr{E}_{\min }$ was sufficient for an accurate determination of $\eta_{\text {nom }}$.) On the other hand, a large cyclotron radius for primary electrons does not have an adverse effect on nominal propellant utilization. Apparently the effect of a large $r_{c}$ is to increase the $\mathscr{E}$ at all values of $\eta$ - that is, to raise the whóle energy-utilization curve shown in figure 2 . Thus, doubling $\mathscr{E}_{\min }$ to obtain $\eta_{\text {nom }}$ could give the same value of $\eta_{\text {nom }}$, regardless of the absolute level of $\mathscr{E}^{\mathscr{E}} \mathrm{min}$ 


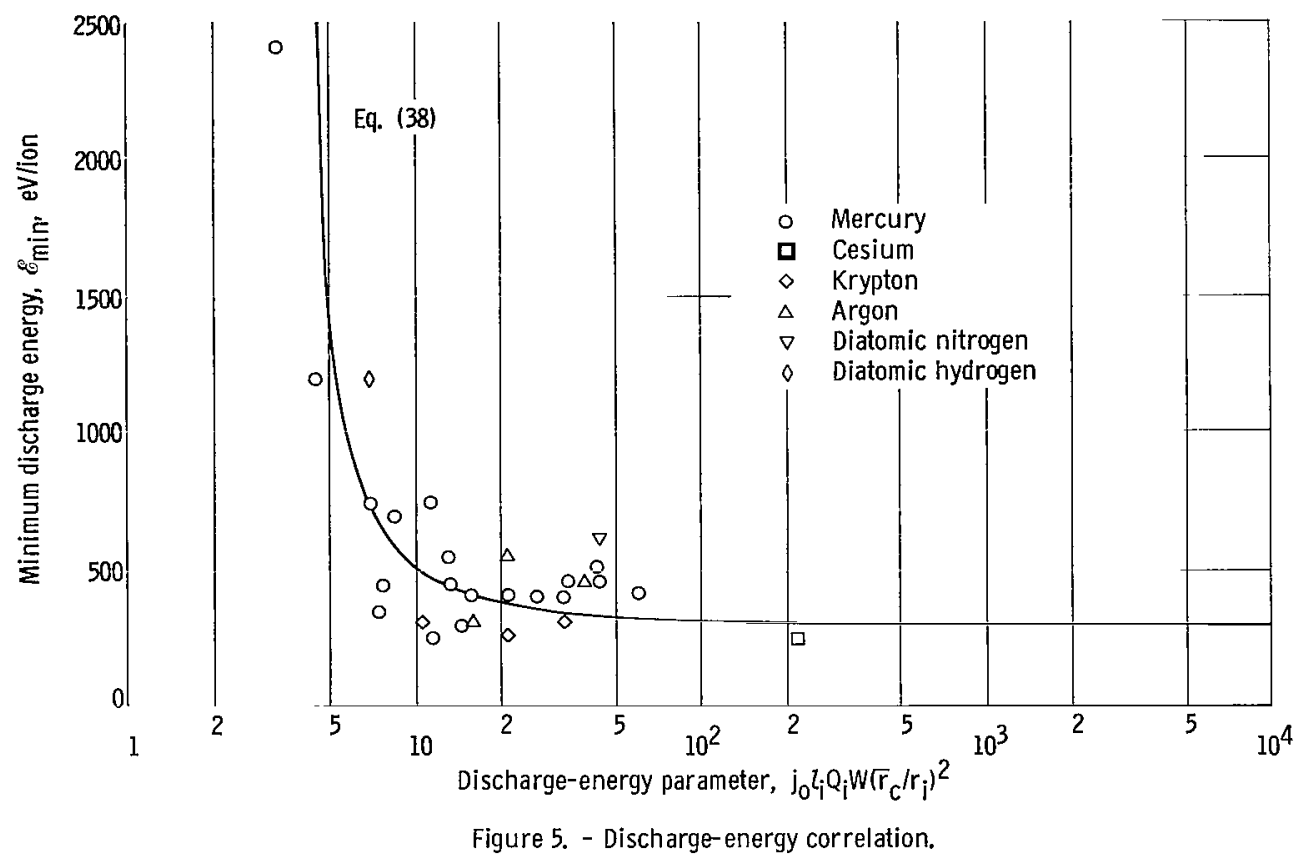

Although the experimental data in figure 4 are lower than the curve of equation (34), they show the same general trend. An interesting aspect of figure 4 is that the nominalpropellant-utilization data at large values of the propellant-utilization parameter level out at a mean value of approximately 0.8 , while the predicted values approach unity. The experimental data of reference 17 show that the primary-electron density drops rapidly as the outer wall, or anode, is approached, contrary to the assumption of uniformity made in the analysis. The neutrals could escape from this outer region with a probability of ionization much lower than the mean value, and unity propellant utilization should be approached at a much slower rate than indicated by equation (34).

The minimum-discharge-energy data are replotted in figure 5, with the omission of data with low discharge potential differences (except for cesium propellant data) and data with large cyclotron radii for the primary electrons. In addition, equation (25) was modified slightly by using $300 \mathrm{eV}$ per ion as the base level of the discharge energy $\mathscr{E}_{0}^{5}:$

$$
E_{\min }=\frac{300}{1-\frac{4\left(\mathrm{r}_{\mathrm{i}} / \overline{\mathrm{r}}_{\mathrm{c}}\right)^{2}}{\mathrm{j}_{\mathrm{o}} \ell_{\mathrm{i}} \mathrm{Q}_{\mathrm{i}} \mathrm{W}}}
$$

The change from 100 to $300 \mathrm{eV}$ per ion cannot be justified with the present poor understanding of ionization-chamber processes, except to say that it gives a better fit to the experimental data. 


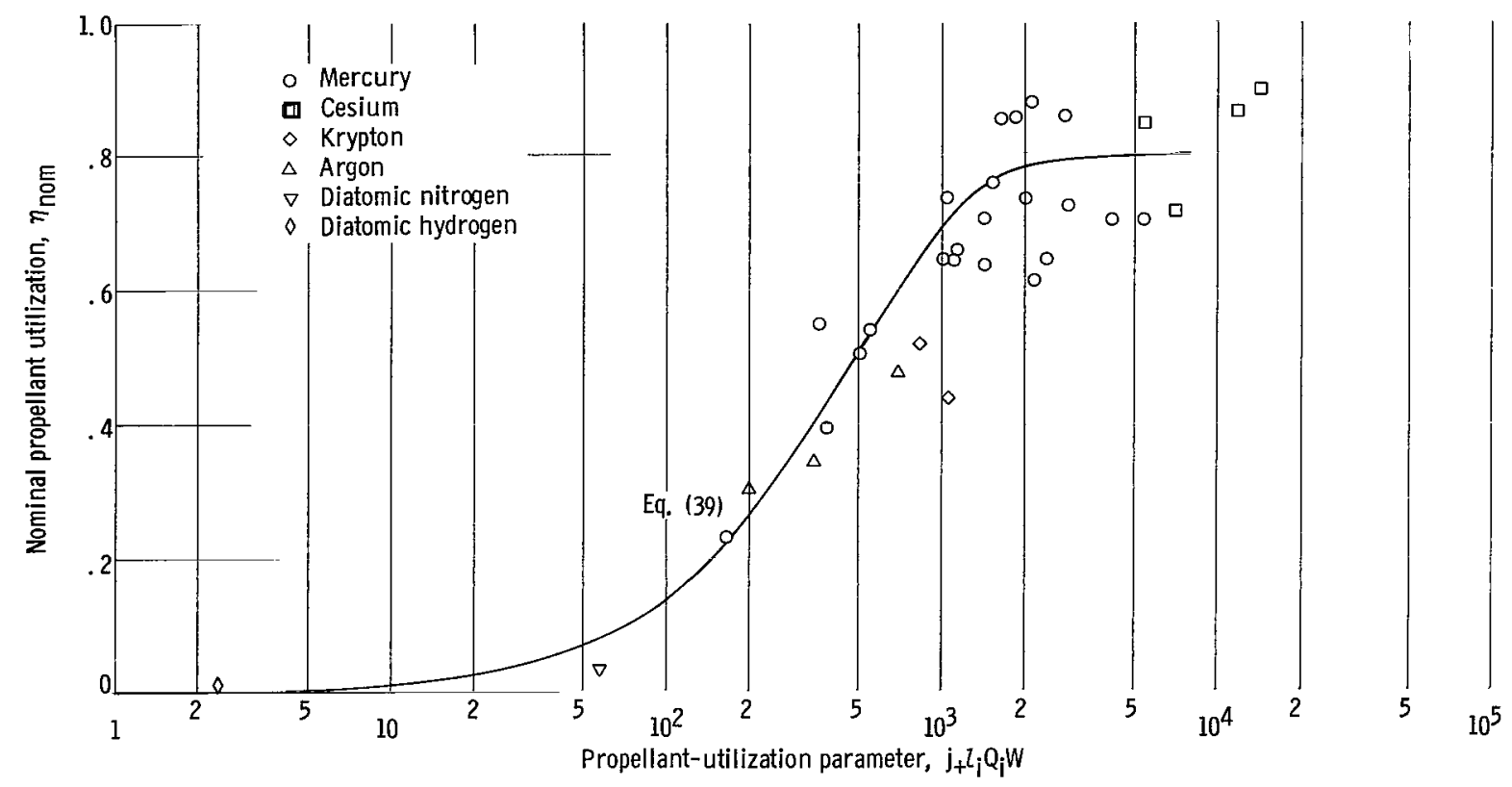

Figure 6. - Propellant-utilization correlation.

With a few exceptions, the experimental correlation is within about \pm 50 per cent of equation (38). This degree of correlation is about as close as can be expected without specifying design details.

The nominal-propellant-utilization data are replotted in figure 6 , with the omission of data with low discharge potential differences (again, except for cesium propellant data). Also, equation (34) was modified by multiplying the right side by a factor of 0.8 to approximate what are thought to be outer-wall effects, and by replacing the constant 0.004 with 0.002 to shift the curve to the right:

$$
\eta_{\text {nom }}=0.8\left[1-\exp \left(-0.002 j_{+} \ell_{i} Q_{i} W\right)\right]
$$

Again, the best justification for these changes is simply the improved fit to the experimental data. The largest percentage discrepancy in figure 6 is for the single point of diatomic nitrogen, where the experimental value is only about one-fourth of the equation value. The only other point below a nominal propellant utilization of 0.2 is for diatomic hydrogen, with the experimental value about 50 percent greater than the equation value. Above a nominal propellant utilization of 0.2 , which is the range of greatest interest, experimental values are within about \pm 20 percent of equation (39). This degree of correlation is again about as close as can be expected without specifying design details.

The assumptions used in the preceding section are, to some extent, validated by the degree of correlation shown in figures 5 and 6 . With the change in $\xi_{0}$ from 100 
to $300 \mathrm{eV} /$ ion, the general agreement between experiment and theory is quite good for the discharge-energy correlation. This agreement does not necessarily mean that the assumed loss mechanisms are responsible for all the variation in discharge energy. For example, operation at high magnetic fields has been recognized for some time as a cause of considerable plasma noise (similar to that measured in ref. 21). The potential fluctuations of this noise would scatter primary electrons, often permitting them to reach the anode without producing ions. Thus the increased discharge energy at low values of the discharge-energy parameter could be the result of either a large plasma potential difference (as assumed), or increased plasma turbulence, or both. The difference in base levels, though, indicates that more than the assumed loss mechanisms are involved.

\section{Application of Correlation to Design}

The highest propellant utilization and the lowest discharge energy that can be conveniently obtained are, of course, desirable for any application. But practical considerations of size, weight, and mechanical complexity usually restrict the performance that is actually obtained. Equations (38) and (39) are fair approximations of the experimental-data correlations shown in figures 5 and 6 . To avoid the region of greatly increased discharge energy, the value of the discharge-energy parameter should be greater than about 10 for almost any application. For the propellant-utilization parameter, a range of values may be of interest. A utilization of about 0.5 might be adequate for either a satellite attitude-control thrustor or a large ground-based ion source. An ion source is not required to operate at a propellant utilization equal to the nominal value, but any large departure from the nominal value is usually wasteful of power or propellant mass. Thus the nominal propellant utilization will be assumed to be approximately synonymous with the operating propellant utilization. For a propellant utilization of 0.5 , then, the propellant utilization parameter should equal, or exceed, a value of about 500. For a large ion thrustor serving as a component of the main propulsion system of a spacecraft, the propellant-utilization parameter should have a value of about 2500 to obtain the high utilization typically required for this application.

Several mathematical expressions are useful for the application of the correlation to design problems. A convenient starting point for a design is to assumed a value for the propellant utilization, and hence for the propellant-utilization parameter. Since $j_{O}$ equals $\mathrm{j}_{\downarrow} / \eta_{\text {nom }}$, part of the discharge-energy parameter can then be calculated:

$$
\mathbf{j}_{\mathrm{o}} \ell_{i} \mathbf{Q}_{i} \mathrm{~W}=\frac{\mathrm{j}_{+} \ell_{i} \mathbf{Q}_{i} W}{\eta_{\text {nom }}}
$$

The ratio of $\bar{r}_{c}$ to $r_{i}$ can be determined from the preceding result, the assumed value for the discharge-energy parameter, and the following identity: 


$$
\frac{\bar{r}_{c}}{r_{i}}=\left[\frac{j_{o} \ell_{i} Q_{i} w\left(\frac{\bar{r}_{c}}{r_{i}}\right)^{2}}{j_{o} \ell_{i} Q_{i} W}\right]^{1 / 2}
$$

The ratio of $r_{c}$ to $\bar{r}_{c}$ is useful for converting between $\bar{r}_{c} / r_{i}$ and $r_{c} / r_{i}$. Dividing equation (37) by equation (26) gives

$$
\frac{\mathrm{r}_{\mathrm{c}}}{\overline{\mathrm{r}}_{\mathrm{c}}}=1.15\left(\frac{\Delta \mathrm{V}_{\mathrm{i}}}{\varphi_{\mathrm{i}}}\right)^{1 / 2}
$$

The sequence of steps to calculate operating conditions for a given value of propellant utilization are as follows:

(1) From assumed $\eta_{\text {nom }}$ and either equation (39) or figure 6, determine $j_{+} \ell_{i} Q_{i} W$.

(2) With $Q_{i}$ and $W$ of assumed propellant, assumed value of $j_{+}$, and $j_{+} \ell Q_{i} W$, calculate $\ell_{i}$.

(3) From assumed ion current and $j_{t}$, determine $r_{i}$.

(4) Using $\eta_{n o m}, j_{+} l_{i} Q_{i} W$, and equation (40), find $j_{0} l_{i} Q_{i} W$.

(5) To maximize $j_{0} l_{i} Q_{i} W\left(\bar{r}_{c} / r_{i}\right)^{2}, \bar{r}_{c}$ should be a maximum. Thus $\Delta V_{i} / \varphi_{i}$ should be slightly above the lower limit of 2.0 , and $r_{c} / r_{i}$ should be slightly below the upper limit of 0.45 . Assume 2.5 and 0.35 as starting values. Note that the $\Delta V_{i} / \varphi_{i}$ and $\mathrm{r}_{\mathrm{c}} / \mathrm{r}_{\mathrm{i}}$ limits were discussed in connection with figure 3 .

(6) From $\varphi_{\mathrm{i}}$ of assumed propellant and $\Delta \mathrm{V}_{\mathrm{i}} / \varphi_{\mathrm{i}}$, find $\Delta \mathrm{V}_{\mathbf{i}}$.

(7) With $r_{i}$ and $r_{c} / r_{i}$, find $r_{c}$.

(8) Using $\Delta V_{i}, r_{c}$, and equation (37), calculate $B$.

(9) From $\Delta V_{i} / \varphi_{i}$ and equation (42), determine $r_{c} / \bar{r}_{c}$.

(10) From $r_{c} / r_{i}$ and $r_{c} / \bar{x}_{c}$ find $\bar{r}_{c} / r_{i}$.

(11) Using $j_{0} \ell_{i} Q_{i} W$ and $\bar{r}_{c} / r_{i}$, calculate $j_{o} \ell_{i} Q_{i} w\left(r_{c} / r_{i}\right)^{2}$.

(12) With $j_{o} l_{i} Q_{i} W\left(\bar{r}_{c} / r_{i}\right)$ and either equation (38) or figure 5 , determine $\mathrm{eV}$ perion.

Starting with a value of 0.5 for $\eta_{\text {nom }}$, this procedure gives a value of 37 for $\mathrm{j}_{\mathrm{o}} \ell_{i} \mathrm{Q}_{\mathrm{i}} \mathrm{W}\left(\overline{\mathrm{r}}_{\mathrm{c}} / \mathrm{r}_{\mathrm{i}}\right)^{2}$. The minimum discharge energy should thus be between about 200 and $500 \mathrm{eV}$ per ion, and the discharge energy near the nominal utilization should be twice as much.

On the other hand, a value of 10 for $\mathrm{j}_{\mathrm{o}} \ell_{i} \mathrm{Q}_{\mathrm{i}} \mathrm{W}\left(\overline{\mathrm{r}}_{\mathrm{c}} / \mathrm{r}_{\mathrm{i}}\right)^{2}$ may be considered sufficient to obtain good performance. This value of 10 and the value of $j_{0} l_{i} Q_{i} W$ from step (2) can be used to find the ratio $\bar{r}_{\mathrm{c}} / \mathrm{r}_{\mathrm{i}}$ from identity (41). With a reasonable value of $4 \varphi_{\mathrm{i}}$ for $\Delta V_{i}, \bar{r}_{c} / r_{i}$, and equation (42), a value of 0.23 is found for $r_{c} / r_{i}$. The ratio 
$r_{c} / r_{i}$ is thus well below the limit of 0.45 . The value of $j_{o} \ell_{i} Q_{i} W\left(\bar{r}_{c} / r_{i}\right)^{2}$ indicates a minimum discharge energy between about 250 and $750 \mathrm{eV}$ per ion. The decrease in $\vec{r}_{c} / r_{i}$ from the preceding example indicates that the magnetic field is almost doubled.

A point demonstrated by these calculations is that there usually is a range of operating conditions that is acceptable from the viewpoint of discharge-energy requirements. If optimum performance is not required, simply assuming values for the ratios $\Delta V_{i} / \varphi_{i}$ and $r_{c} / r_{i}$ will fix the particular operating point within this range. For optimum performance, however, considerable trial-and-error work must be done. For example, most of the variations evaluated in reference 29 are within an acceptable range, as far as the performance correlation is concerned. The range of performance obtained in reference 29, though, is ample evidence that an optimum design is not completed with the performance-correlation calculations. The use of the performance correlation should constitute only the first step in obtaining a near-optimum design.

\section{Additional Design Constraints}

There are a number of limits to observe in applying the results of the performance correlations. The most basic limit probably is that the ionization chamber is only one of several thrustor or ion-source components, and an operating point has to be reasonable for all the components - not just the ionization chamber. Thus the ion-beam-current density $j_{+}$selected for the ionization chamber should not exceed the space-charge limit for the accelerator system; the combination of total ion-beam current, total discharge power, and discharge potential difference should not require a cathode emission beyond what is available.

The ionization-chamber limits for $r_{c} / r_{i}(\leq 0.45)$ and $V_{i} / \varphi_{i}(\geq 2.0)$ have been mentioned. The different mode of operation that can be obtained at low discharge potential differences with cesium, or presumably with any other low-ionization-potential propellant, has also been mentioned. The use of atoms with metastable states might be expected to extract more useful energy from the secondary electrons, but the data for argon in figure 5 show no such advantage. Diatomic gases have the problem of monatomic versus diatomic ions. Although the diatomic-gas data presented herein were for predominantly diatomic-ion beams, data in references 5 and 6 indicate that ion beams should be mostly monatomic for propellant utilizations of 0.5 or higher. Molecules more complex than diatomic tend to fragment excessively (refs. 31 and 32). Also, the many possible modes of excitation for complex molecules tend to make the estimation of secondary-electron temperature very difficult.

There are also geometric limits to observe. The natural solution to many ionsource problems apparently is to increase the ionization-chamber length $\ell_{i}$. However, 
as shown in reference 29 , increasing $\ell_{i}$ is not an unmixed blessing. Beyond a certain length-to-radius ratio $\ell_{\mathrm{i}} / \mathrm{r}_{\mathrm{i}}$, the discharge energy increases instead of decreases. This effect does not disprove the correlation of figure 5 , but is simply one of the effects included within the data spread. All but two of the points in figures 5 and 6 were within the $\ell_{i} / r_{i}$ range from 1.5 to 3.3. These two additional points were at 0.7 and 5.0. The lack of larger values of $\ell_{i} / r_{i}$ is particularly important near the critical value of 10 for the discharge-energy parameter, where the benefits are presumably greatest.

An interesting geometrical variation was used for part of the investigation reported in reference 17. Only the central quarter of the accelerator area was used, although the anode remained its original diameter. This modification was accomplished by covering holes in the upstream accelerator grid for all except the center half of the radius. From other current-density profiles, it is estimated that only about half of the ions that reached the accelerator end of the ionization chamber were lost as the result of covering accelerator holes. On this basis the discharge energy per beam ion should have been, and was, about double the uncovered-accelerator value. The area for neutral escape, though, was reduced by a factor of 4 . Thus the propellant utilization was substantially improved. Using only the central portion of the accelerator grids can therefore serve to increase the propellant utilization, but only at the cost of an increased discharge energy.

A few closing comments on design of an electron-bombardment ion source may be useful. Operation is generally best with a magnetic field that decreases in strength continuously in the downstream direction. The best range of minimum-to-maximum fieldstrength ratio is from 0.4 to 0.8 . For the cathode, more emitting area generally decreases the lower stable limit for $\Delta \mathrm{V}_{\mathrm{i}}$. Area that is associated with the cathode and its support, other than emitting, should be minimized. The mode of propellant introduction into the ionization chamber is best solved with some degree of trial and error. For very low ion-current densities, the performance is generally best with the propellant introduced near the cathode. For high ion-current densities, a more uniform propellant introduction is generally best. If parallel plates with hexagonal arrays of holes are used for an accelerator system, the upstream grid should have not more than about 50-percent blockage, and a thickness not more than about a third of the hole diameter. For the downstream grid, the hole diameter can be decreased slightly and the thickness increased substantially without adverse effects.

\section{CONCLUDING REMARKS}

The minimum discharge energy (per ion) $\mathscr{E}^{6}$ min and the propellant utilization obtained at a discharge energy of $2 \varepsilon_{\text {min }}$ (the nominal propellant utilization $\eta_{\text {nom }}$ ) were selected as giving, by themselves, a fair representation of overall ionization-chamber 
performance. The generalizing parameters derived to correlate experimental data were $\mathrm{j}_{\mathrm{o}} \ell_{i} \mathrm{Q}_{\mathrm{i}} \mathrm{W}\left(\overline{\mathrm{r}}_{\mathrm{c}} / \mathrm{r}_{\mathrm{i}}\right)^{2}$ (the discharge-energy parameter) and $\mathrm{j}_{+} \ell_{i} \mathrm{Q}_{\mathbf{i}} \mathrm{W}$ (the propellant-utilization parameter). The units are those of the mks system except for $Q_{i}$ (in $\pi a_{0}^{2}$ units) and $\mathrm{W}$ (in $\mathrm{amu}$ ). Using these generalizing parameters correlates the experimental data within about \pm 50 percent for minimum discharge energy and about \pm 20 percent for nominal propellant utilization. The minimum discharge energy remains nearly constant at about $300 \mathrm{eV}$ per ion over most of the range of discharge-energy parameter, but rises rapidly at values less than about 10 . The nominal propellant uitlization is near 0.8 at values of the propellant-utilization parameter above about 2000 , but decreases at smaller values.

Two of the limits on the correlations are: the discharge potential difference should be at least 2.0 times the first ionization potential of the propellant, and the cyclotron radius of the primary electrons should be less than about 0.45 times the ionizationchamber radius. The correlations are applicable to most atomic propellants. The areas of uncertainty concern low-ionization-potential propellants and diatomic gases. According to the correlations, performance is generally improved for long ionization chambers. Extensive data, though, are not available for length-to-radius ratios greater than about 3 . Also, the effect of length-to-radius ratio is probably one of the secondary effects that are masked by the data spread of the correlation. This data spread appears to be due to variations in design details, other than those included in the correlating parameters.

\section{Lewis Research Center,}

National Aeronautics and Space Administration, Cleveland, Ohio, July 15, 1965. 


\section{APPENDIX - SYMBOLS}

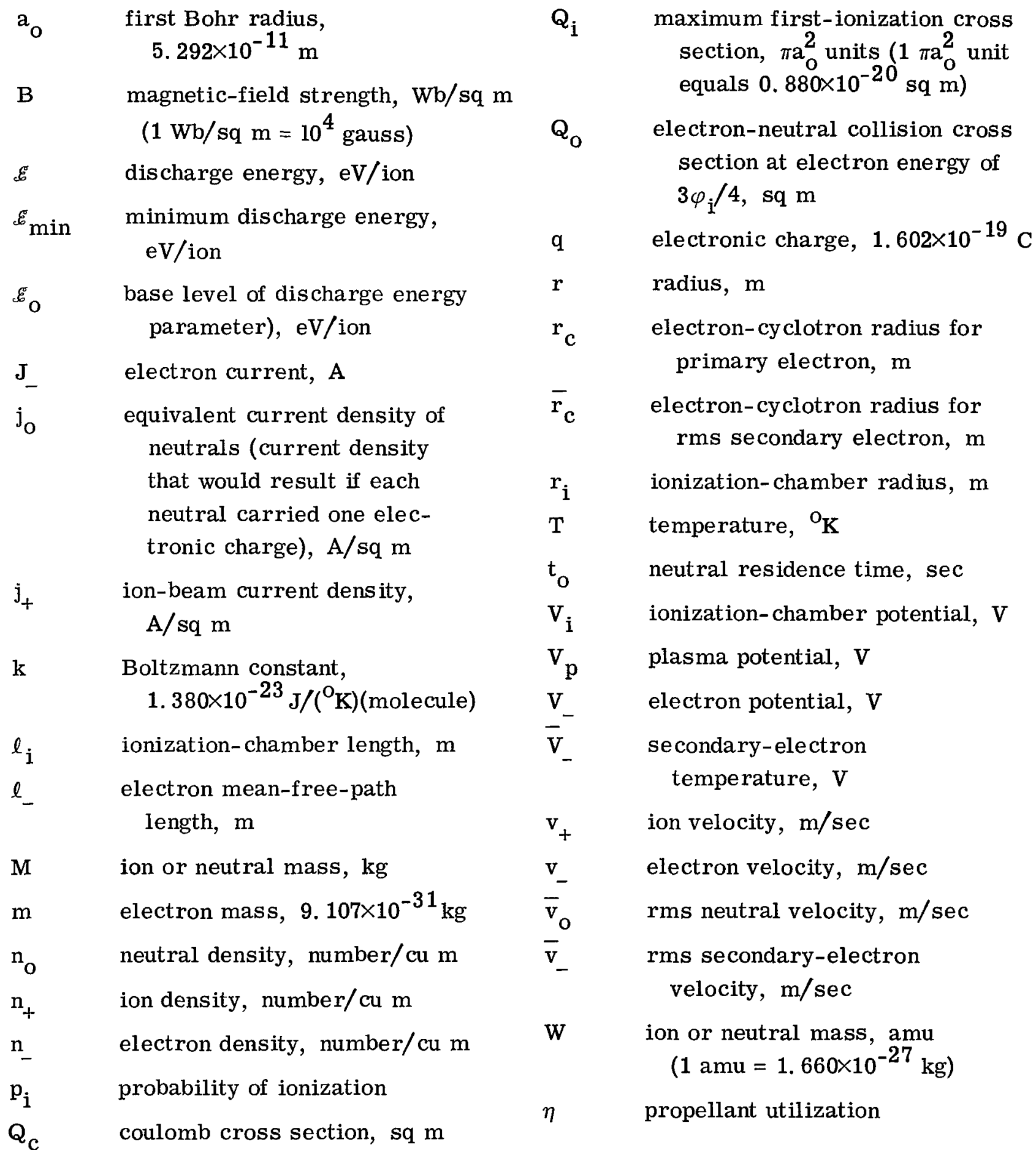


$\eta_{\text {nom }} \quad$ nominal propellant utilization (where $\mathscr{E}$ equals twice $\mathscr{E}_{\text {min }}$ )

$\sigma_{0} \quad$ plasma conductivity with no magnetic field, $\mathrm{A} / \mathrm{V}-\mathrm{m}$

$\sigma_{\perp} \quad$ plasma conductivity normal to magnetic field, $\mathrm{A} / \mathrm{V}-\mathrm{m}$ $\tau$ - mean electron collision time, sec

$\varphi_{i} \quad$ first ionization potential of atom or molecule, eV

$\omega$ electron-cyclotron frequency, $\mathrm{rad} / \mathrm{sec}$ 


\section{REFERENCES}

1. Kaufman, Harold R.: An Ion Rocket with an Electron-Bombardment Ion Source. NASA TN D-585, 1961.

2. Kaufman, Harold R.; and Reader, Paul D.: Experimental Performance of Ion Rockets Employing Electron-Bombardment Ion Sources. Progress in Astronautics and Rocketry. Vol. 5 - Electrostatic Propulsion, D. B. Langmuir, E. Stuhlinger, and J. M. Sellen, Jr., eds. Academic Press, Inc., 1961, pp. 3-20.

3. Livingston, M. Stanley; Holloway, M. G.; and Baker, C. P.: A Capillary Ion Source for the Cyclotron. Rev. Sci. Instr., vol. 10, no. 2, Feb. 1939, pp. 63-67.

4. Finkelstein, A. Theodore: A High Efficiency Ion Source. Rev. Sci. Instr., vol. 11, no. 3, Mar. 1940, pp. 94-97.

5. Meyerand, Russell G., Jr. ; and Brown, Sanborn C.: High-Current Ion Source. Rev. Sci. Instr., vol. 30, no. 2, Feb. 1959, pp. 110-111.

6. Moak, C. D.; Banta, H. E. ; Thurston, J. N.; Johnson, J. W. ; and King, R. F. : Duo Plasmatron Ion Source for Use in Accelerators. Rev. Sci. Instr., vol. 30, no. 8, Aug. 1959, pp. 694-699.

7. Kerslake, William R. : Accelerator Grid Tests on an Electron-Bombardment Ion Rocket. NASA TN D-1168, 1962.

8. Kerslake, William R. ; and Pawlik, Eugene V.: Additional Studies of Screen and Accelerator Grids for Electron-Bombardment Ion Thrustors. NASA TN D-1411, 1963.

9. Kerslake, William R. : Charge-Exchange Effects on the Accelerator Impingement of an Electron-Bombardment Ion Rocket. NASA TN D-1657, 1963.

10. Milder, Nelson L.; and Kerslake, William R. : Evaluation of Filament Deterioration in Electron-Bombardment Ion Sources. NASA TN D-2173, 1964.

11. Kerslake, William R. : Cathode Durability in the Mercury Electron-Bombardment Ion Thrustor. Paper No. 64-683, AIAA, 1964. (See also NASA TM X-52037, 1964.)

12. Kerslake, William R. : Preliminary Operation of Oxide-Coated Brush Cathodes in Electron-Bombardment Ion Thrustors. NASA TM X-1105, 1965.

13. Speiser, R. C. ; Kilpatrick, W. D.; and Reid, G. C.: Study of a Gas Discharge Cesium Ion Source. NASA CR-52366, 1963.

14. Reader, Paul D.: An Electron-Bombardment Ion Rocket with a Permanent Magnet. Paper No. 63031, AIAA, 1963. (See also Astronaut. and Aerospace Eng., vol. 1 , no. 9 , Oct. 1963 , p. 83. ) 
15. Reader, Paul D.: Scale Effects on Ion Rocket Performance. ARs J., vol. 32, no. 5, May 1962, pp. 711-714. (See also Paper No. 61-87-1781, ARS, 1961.)

16. Pawlik, Eugene V.; and Nakanishi, Shigeo: Experimental Evaluation of Size Effects on Steady-State Control Properties of Electron-Bombardment Ion Thrustor. NASA TN D-2470, 1964.

17. Reader, Paul D.: The Operation of an Electron-Bombardment Ion Source with Various Gases. First Int. Conf. on Elect. and Ion Beam Science and Tech., Robert Bakish, ed., John Wiley \& Sons, Inc., 1965, pp. 925-935. (See also NASA TM X-52006, 1964.)

18. Strickfaden, W. B.; and Geiler, K. L. : Probe Measurements of the Discharge in an Operating Electron Bombardment Engine. Paper No. 63056, AIAA, 1963. (See also NASA CR-50623, 1963.)

19. Domitz, Stanley: Experimental Evaluation of a Direct-Current Low-Pressure Plasma Source. NASA TN D-1659, 1963.

20. Kaufman, Harold R. : Electron Diffusion in a Turbulent Plasma. NASA TN D-1324, 1962.

21. Spitzer, Lyman, Jr.: Physics of Fully Ionized Gases. Intersci. Pub., Inc., 1956.

22. Brown, Sanborn C.: Basic Data of Plasma Physics. John Wiley \& Sons, Inc., and Technology Press of M. I. T., 1959.

23. Bohm, David: Minimum Ionic Kinetic Energy for a Stable Sheath. The Characteristics of Electrical Discharges in Magnetic Fields, A. Guthrie and R. K. Wakerling, eds. , McGraw-Hill Book Co., Inc., 1949, pp. 77-86.

24. Kieffer, L. J.: A Compilation of Critically Evaluated Electron Impact Ionization Cross Section Data for Atoms and Diatomic Molecules. JILA Rept. No. 30, Univ. of Colorado, 1965.

25. Reader, Paul D.: Experimental Performance of a 50-Centimeter Diameter ElectronBombardment Ion Rocket. Paper No. 64-689, AIAA, 1964. (See also NASA TM X-52042, 1964.)

26. Barcatta, F. A.; Reid, G. C.; Sohl, G.; Spieser, R. C. ; and Worlock, R. M. : Ion Rocket Engine System Research and Development. NASA CR-54067, 1964.

27. Milder, Nelson L. : Comparative Measurements of Siingly and Doubly Ionized Mercury Produced by Electron-Bombardment Ion Engine. NASA TN D-1219, 1962.

28. Reader, Paul D. : Experimental Effects of Propellant-Introduction Mode on ElectronBombardment Ion Rocket Performance. NASA TN D-2587, 1965. 
29. Reader, Paul D.: Investigation of a 10-Centimeter-Diameter Electron-Bombardment Ion Rocket. NASA TN D-1163, 1962.

30. Evans, Robley D.: The Atomic Nucleus. McGraw-Hill Book Co. , Inc., 1955, p. 659 .

31. Milder, Nelson L. : Fragmentation of Anthracene in an Electron-Bombardment Ion Source. NASA TN D-2592, 1965.

32. Byers, David C. ; Kerslake, William R. ; and Grobman, Jack: Experimental Investigation of Heavy-Molecule Propellants in an Electron-Bombardment Thrustor. NASA TN D-2401, 1964. 
"The aeronautical and space activities of the United States shall be conducted so as to contribute. . . to the expansion of human knowledge of phenomena in the atmospbere and space. The Administration shall provide for the widest practicable and appropriate dissemination of information concerning its activities and the results thereof."

-National Aeronautics and Space Act of 1958

\section{NASA SCIENTIFIC AND TECHNICAL PUBLICATIONS}

TECHNICAL REPORTS: Scientific and technical information considered important, complete, and a lasting contribution to existing knowledge.

TECHNICAL NOTES: Information less broad in scope but nevertheless of importance as a contribution to existing knowledge.

TECHNICAL MEMORANDUMS: Information receiving limited distribution because of preliminary data, security classification, or other reasons.

CONTRACTOR REPORTS: Technical information generated in connection with a NASA contract or grant and released under NASA auspices.

TECHNICAL TRANSLATIONS: Information published in a foreign language considered to merit NASA distribution in English.

TECHNICAL REPRINTS: Information derived from NASA activities and initially published in the form of journal articles.

SPECIAL PUBLICATIONS: Information derived from or of value to NASA activities but not necessarily reporting the results of individual NASA-programmed scientific efforts. Publications include conference proceedings, monographs, data compilations, handbooks, sourcebooks, and special bibliographies.

Details on the availability of these publications may be obtained from:

SCIENTIFIC AND TECHNICAL INFORMATION DIVISION

NATIONAL AERONAUTICS AND SPACE ADMINISTRATION

Washington, D.C. 20546 